\title{
Progress of Astronomy in India: A Scientometric Study base on paper published during 1991 - 1995 and 2011 - 2015
}

\author{
Vijay Kumar Rai ${ }^{1, \star}$, K. P. S. Senger ${ }^{2}$, and Rajesh K Lohiya ${ }^{3}$ \\ ${ }^{1}$ IUCAA, Ganeshkhind Pune, India \\ ${ }^{2}$ IMTECH, Chandigarh, India \\ ${ }^{3} \mathrm{NEERI}$, Nagpur, India
}

\begin{abstract}
Astronomy is the oldest of the natural sciences. It is well known that the period covering about seven centuries, from Aryabhata to Bhaskaracharya II (c. 476-1150), was the golden age of Indian astronomy. In the present study, we identify research trends and the growth of knowledge in the field of astronomy science research. The study is an essential tool to measure the scientific publications in the field. This study analyses the publications of astronomy research in India published during 1991-1995 and 2011-2015. The study assesses how astronomy progresses in India and its impact is reflected in the science citation index over the period 1991-1995 and 2011-2015. The publication output has been analyzed using quantitative and qualitative indicators, such as progress in research article publishing, change in authorship patterns, citation patterns during 19911995 and 2011-2015, articles that received the most citations, and international collaboration with other countries. Further, the study investigated highly prolific authors and highly preferred journals. The author is from one of the FORSA institutes, therefore the study includes a separate ranking for FORSA institutes in term of output as well citations.
\end{abstract}

\section{Introduction}

The science of astronomy has fascinated humans from the beginning of time. In the early stages of the evolution of ideas, we found conceptions of the Mother Earth being entertained by Father Sky to nourish her and create life. The journey from there to new satellites, such as ASTROSAT, has been fascinating.. In India, astronomy has a long and has a glorious past. There is documented evidence that astronomy was studied in India from the Vedic times dating back to $1500 \mathrm{BC}$, and then nurtured in the fifth and sixth century AD by the greats like Aryabhatta, Varahamihira, and Brahmagupta.. The advent of telescopes shifted the leadership in astronomy studies from Asia and the Middle East to Europe.

There are numerous institutes in India which are doing frontline research in astronomy and astrophysics, and there are even several telescope facilities now available. They include solar telescopes, radio telescopes, optical telescopes and others. India is marching ahead in astronomy research aided by ground-based, balloon-borne and satellite facilities. The future of astronomy in India is bright and the next decade promises that there will be substantial contributions coming from India.

^e-mail: vkrai@iucaa.in ORCID: 0000-0003-4548-403X 
In the early 1980s, due to the proliferation of information, library professionals working in the Institutes where astronomy is one of the main areas of research, felt the need to come together and form a forum, which can act as a springboard for sharing and exchanging information. The sheer necessity brought all likeminded astronomy librarians in the country together. The Forum for Resource Sharing in Astronomy and Astrophyics (FORSA) was launched on July 29, 1981, with a vision and mission to share resources held in each library. At present, there are eleven Institute members: Aryabhatta Research Institute of Observational Sciences (ARIES), SN Bose National Centre for Basic Science, CASA-OU, Harish-Chandra Research Institute (HRI), Indian Institute of Astrophysics (IIA), InterUniversity Centre for Astronomy and Astrophysics (IUCAA), National Centre for Radio Astronomy (NCRA), Physical Research Laboratory (PRL), Raman Research Institute (RRI), Saha Institute of Nuclear Physics (SNIP), and Tata Institute of Fundamental Research (TIFR) [1].

Research trends and growth of knowledge in a scientific field can be identified by using essential tools to measure the scientific publications in the field. There are various quantitative methods and techniques to use for measuring the impact of scientific work. It has become essential to adopt standard operating procedures and to measure the research work using various methods from historic bibliometric to modern scientometric. There are tools to measure the science and technology, most notably, for published papers in referred journals the scientometric technique is applied. Scientific research productivity usually culminates in scientific writing, which leads to research publications. Scientific literature is considered to be a reliable medium for promoting national and professional interest in one's field of specialization.

\section{Past Studies}

Astronomy and astrophysics have been subject to a number of scientometric studies in the US and Europe, but in India only a few studies have been conducted. These include a scientometric study of the doctoral theses of aphysical research laboratory done by Nistha Anilkumar [2]. Barve and Gopal-Krishna(2002)[3] found that there is a steady increase in the number of observational papers published by Indian radio astronomers. They found a steep rise in the number appearing as "rapid publication."

In the productivity of Indian Telescopes study by Meera and Manjunath, [4] they examined the productivity of modern Indian telescopes that were installed after India attained independence from colonial rule. The study found that 1,643 papers were published using Indian telescopes. The highest number of citations is associated with the GMRT (Radio Telescope) papers. The first international publication from India was in the "Monthly Notices of Royal Astronomical Society" in 1906. India ranked fifteenth in the Global Aurora research with 182 publications and 1559 papers from astronomy and astrophysics (Dwivedi, 2016)[5]

A scientometric study of astronomy research in IUCAA done by Vijay Kumar Rai and K P S Senger found that with a collaborative approach in research, several papers received an extraordinary number of citations and many papers were published in high impact journals[6]. A similar study of visiting associates of IUCAA found that there is a collaborative approach in research among them, and the degree of collaboration is quite high [7]. Dutta and Rath (2013) studied cosmology research in India and found that it is a mainly collaborative effort with the USA and other European countries. The study found that there are some exorbitantly large teams with more than one hundred members, and sometimes as many as 715. Almost all core journals are published by the USA and European countries and possess relatively high impact factor. The study found that individually IUCAA, IITs and Jadavpur University are the top three affiliating institutions [8]. 


\section{Objectives of Study}

The primary aims of the present study are to analyze the broad characteristic features of the publication output of Indian Astronomy during 1991-1995 and 2011-2015, using quantitative and qualitative indicators. In particular, the study focuses on the growth of research output, citation impact, international collaboration, communication channels preferred, and contribution author and highly prolific author.

\section{Source Database and Methodology}

The study is based on the publication data derived from Web of Science Core Collection. The study is limited to the period of 1991-1995 and 2011-2015.

To retrieve data an advance strategy was adopted. First I applied the term India for Author Address and limited the search results to the period 1991-95 and 2011-2015 respectively. The results which I got, I refine with the Subject Category "Astronomy and Astrophysics."

The study periods, 1991-1995 and 2011-2015 have been chosen purposefully to understand the shift in the research output of Indian astronomy. There has been a substantial expansion in the information technology (IT) infrastructure, budget and researchers in later years. The data was analyzed as per the objectives of the study after the validation.

\section{Data Analysis and Interpretation}

To identify research trends and the growth of knowledge in astronomy and astrophysics, it is essential to measure the scientific publications in the field. This study analyses the publications of astronomy research in India published during 1991-1995 and 2011-2015. Study analyses the production activity, distribution of journals, citation analysis, and international collaborations.

\subsection{Publication Activity}

The Indian astronomy and astrophysics research output has increased three folds during 2011-2015 in comparison to 1991-1995. During 1991-95, a total of 1987 articles were published and in 201115 this jumped to 5186, which is 2.61 times higher. There is no definite pattern of literature growth ascertained from the data, at least for the years of 1991-95. The study finds that the research output for the period of 2011-2015 is an unexpected decline, but there was a growth of publication observed for the year of 2016 .

Indian researchers published a total of 1987 papers, with an average number of publications per year. This number was 397 during 1991-1995. During 2011-15, the maximum number of articles published was in 2011 (1175) and the minimum was in 2015 (893), with an average of 1037 as shown in Table 1. The reason for the decline in the number of articles published is yet to be ascertained. Observation indicates that astronomy articles publication does not have a particular pattern.

\subsection{Distribution of journals}

During 1991-95, 1640 articles were published in peer-reviewed journals and the rest published in a note, review, individual proceedings, editorial, etc. Out of 1640, 38 articles were published either in proceedings series or book series. Most of the articles were published in international journals. These 


\section{Paper Published during 1991-95 and 2011-15}

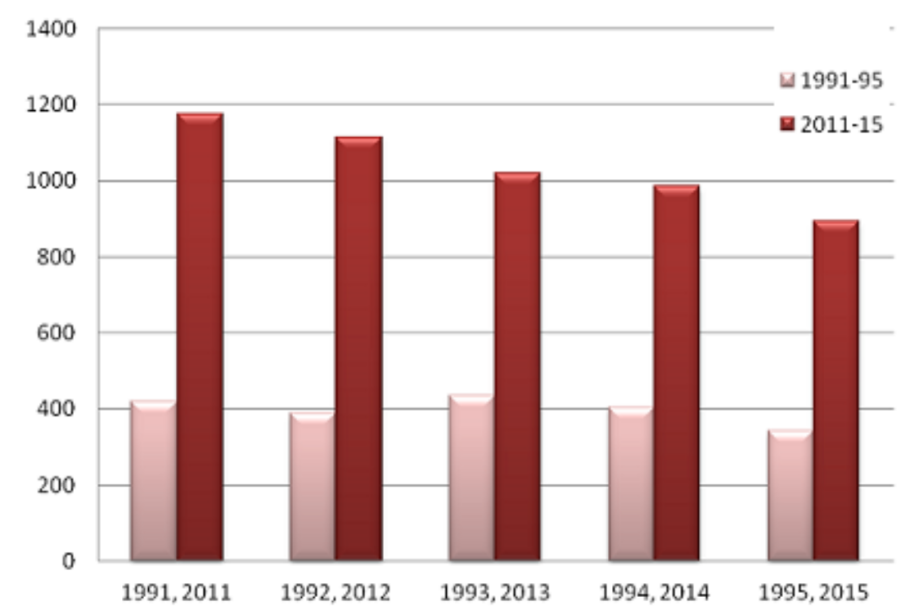

Figure 1. Paper Published during 1991-95 and 2011-15

Table 1. Papers Published and Growth

\begin{tabular}{|c|c|c|c|c|}
\hline Year & No. of Papers & Year & No. of Papers & Growth \\
\hline 1991 & 420 & 2011 & 1175 & $280 \%$ \\
\hline 1992 & 389 & 2012 & 1113 & $286 \%$ \\
\hline 1993 & 434 & 2013 & 1019 & $235 \%$ \\
\hline 1994 & 403 & 2014 & 986 & $245 \%$ \\
\hline 1995 & 341 & 2015 & 893 & $262 \%$ \\
\hline Total & 1987 & & 5186 & $261 \%$ \\
\hline
\end{tabular}

articles were published in 51 journals. Out of these 51 journals, there are only 3 Indian journals, but the highest numbers of articles (252) were published in the Indian Journal of Radio Space Physics. The journals Astrophysics and Space Science (219), Physical review D (208), Physics Letters B (206), Astrophysical Journal (136) published more than 100 articles each. Four journals published between 50-100 articles, 11 journals published between 10-15 articles and 31 journals published ten or fewer articles. While the highest number of papers were published in the Indian Journal of Radio Space Physics, most of the papers were published in well-known international journals.

A total of 4581 articles were published in peer-reviewed journals during 2011-15, including 42 articles published in proceedings and the rest published in correction, editorial material, news item, book chapter, biographical item, etc. The highest numbers of articles were published in Physical Review D (1067). MNRAS (515), Astrophysics and Space Science (490), Physics Letters B (419), and Astrophysical Journal (385) were among the top five journals chosen by researchers to publish articles. These top 5 journals published $57 \%$ of the total articles published by Indian researchers. Four journals published between 100-250 articles. Nine journals published between 50-100 articles. 13 journals published between 10-49 articles. 21 journals published less than 10 articles.

It shows that Indian researchers prefer to publish their papers in International journals with high impact factors. 


\subsection{Citations Analysis}

It is clear that Indian astronomers published their papers in internationally known and high impact factor journals. Therefore, Indian astronomy research articles are well cited. As shown in Table 3, during 1991-95, the highest number of papers (859) were cited between 1-10 times. However, 341 papers were not cited even once. One article is cited 1043 times. During 2011-15, 2779 papers are cited between 1-10 times and 1266 papers are cited between 11-50 times.

Table 2. Citation Analysis

\begin{tabular}{|c|c|c|}
\hline $\begin{array}{c}\text { Citation } \\
\text { received } \\
0\end{array}$ & $\begin{array}{c}\text { Paper Published } \\
1991-1995 \\
314\end{array}$ & $\begin{array}{c}\text { Paper Published } \\
2011-2015 \\
453\end{array}$ \\
\hline $1-10$ & 859 & 2779 \\
\hline $11-50$ & 500 & 1266 \\
\hline $51-100$ & 83 & 122 \\
\hline $101-200$ & 25 & 29 \\
\hline $201-300$ & 4 & 7 \\
\hline $301-400$ & 1 & 3 \\
\hline $401-800$ & 1 & 3 \\
\hline $801+$ & 1 & 4 \\
\hline
\end{tabular}

There are two papers which are cited 4072 times, and one article is cited 3857 times. Citations have consistently increased every year no matter the number of articles published that particular year. There were 1987 articles published during 1991-95. These 1987 papers received 4052 citations in during this period, but overall these papers received 24326 citations until 2017. The average citation per paper was 14.10 and the h-index 68. It was found that the number of publications decline but the number of citations increased for the articles published during 2011-15. Even though a minimum number of articles were published in 2015 , these papers got maximum citations. The average citations per paper is 14.56 and the $\mathrm{h}$-index is 83 . The cumulative citations graph shows that citations for these papers increased every year and still counting.

\subsection{International Collaboration}

Indian astronomy research progresses with international collaboration. During 1991-1995, Indian researchers collaborated with researchers from about 50 countries; however, in 2011-2015 collaboration reached 89 countries. There were no major group or project collaborations in 1991-1995. Indian astronomy researchers showed significant progress in collaborative research at an international level during 2011-2015. Even Indian research is part of many international projects like CMS Collaboration (141), D0 Collaboration (83), BABAR Collaboration (78), BELLE Collaboration (73), ATLAS Collaboration (47), PLANCK Collaboration (41), ALICE Collaboration (39), LIGO SCI Collaboration (22), and VIRGO Collaboration (19).

During 1991-1995 USA (261), Germany (116), Italy (110), France (84), and Switzerland(74) were the among the top five countries which are preferred by researchers for collaboration. During 2011-2015, England and Russia were among the top five countries. Researchers from USA (1590), Germany (1120), France (867), England (851) and Russia (808) collaborated the most with Indian researchers. 


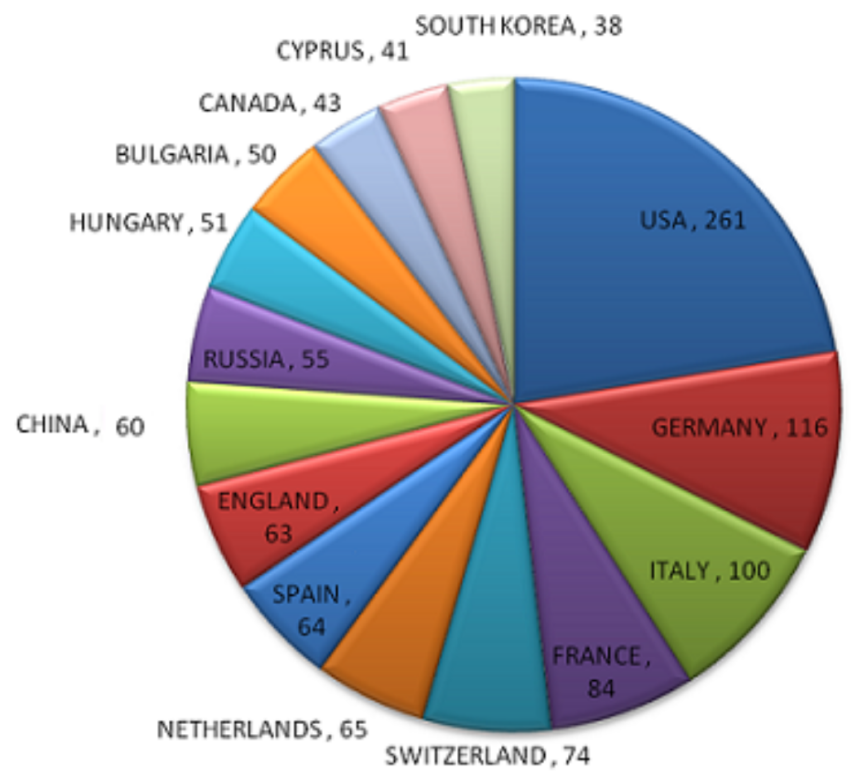

Figure 2. International Collaboration with Top 15 countries

\subsection{Highly Productive Author}

The study has identified 41 Indian authors who have produced the most papers during 1991-1995. Out of these 41 authors, 8 authors produced between 46-53 papers and these papers were published by a small group of authors. All these 8 authors are affiliated with TIFR, Mumbai. Among them, S C Tonwar (53) and V K Gupta (51) produced the most papers. However, K P Singh (22) from the TIFR is the most prolific author, followed by B M Reddy, U Sarkar and R P Singh who published 21 papers each.

The study finds that during 2011-2015 collaborative research was a trend, and the result is scores of collaborations. In total, 66 collaboration groups were found. The top five group authors produced 599 papers.

Chakrabory, S(61) from IUCAA, Chakrabarti, SK(59) from Indian Ctr Space Phys, Subrahmanyan, R(57) from RRI, Kumar S(55) from BITS Pilani, Banerjee, S(52) from IIT Jodhpur were the top five authors.

\section{FORSA Institutes Output Analysis}

FORSA institutes have astronomy research as one of its core research areas. The study analyzed output of FORSA institutes separately. Output of TIFR includes NCRA output as well. Two institutes, UPSO and MRI (1991-1995) were renamed Aryabhatta Research Institute of observational sciencES (ARIES) and Harish Chandra Research Institute during 2011-2015 respectively.

\subsection{Publication Activity}

Among the FORSA institutes SN Bose NCBS and HRI have shown maximum growth but published minimum papers. All FORSA institutes published more than a hundred papers during 2011-15 com- 
pared to three institutes during 1991-95. Cumulative output of FORSA institute is $329.44 \%$ higher during 2011-15.

Table 3. Paper Published by FORSA Institute

\begin{tabular}{|c|c|c|c|}
\hline $\begin{array}{c}\text { FORSA } \\
\text { Institutes }\end{array}$ & $\begin{array}{c}\text { Paper Published } \\
1991-1995\end{array}$ & $\begin{array}{c}\text { Paper Published } \\
2011-2015\end{array}$ & $\begin{array}{c}\text { Growth of } \\
\text { Publications }\end{array}$ \\
\hline TIFR & 368 & 929 & $252.45 \%$ \\
\hline IIA & 213 & 409 & $192.02 \%$ \\
\hline PRL & 155 & 309 & $199.35 \%$ \\
\hline IUCAA & 90 & 514 & $571.11 \%$ \\
\hline RRI & 50 & 225 & $450.00 \%$ \\
\hline UPSO now ARIES & 46 & 207 & $450.00 \%$ \\
\hline SINP & 30 & 347 & $1156.67 \%$ \\
\hline MRI now HRI & 9 & 129 & $1433.33 \%$ \\
\hline SN Bose NCBS & 7 & 120 & $1714.29 \%$ \\
\hline
\end{tabular}

FORSA institutes produced around $48 \%$ of total papers published by Indian astronomers during 1991-95 and it reached 61\% during 2011-15. It shows that FORSA institutes are more focused on astronomy research than other institutes.

\subsection{Citation analysis}

TIFR published the maximum numbers of papers. Citations received and average citations per year by TIFR papers are also the maximum during 1991-95. Papers published by FORSA institutes during 1991-95 have received 18300 citations up to June 2017 with the average citations per item 20.09. The highest citations totaling 1605 were received in 1996 for these papers, and then the number of citations declines gradually. One paper published in 1994 got 1044 citation, and 20 articles published during this period got 100 or more citations. Cumulative citations increased until 1996 for the papers published during 1991-95. 


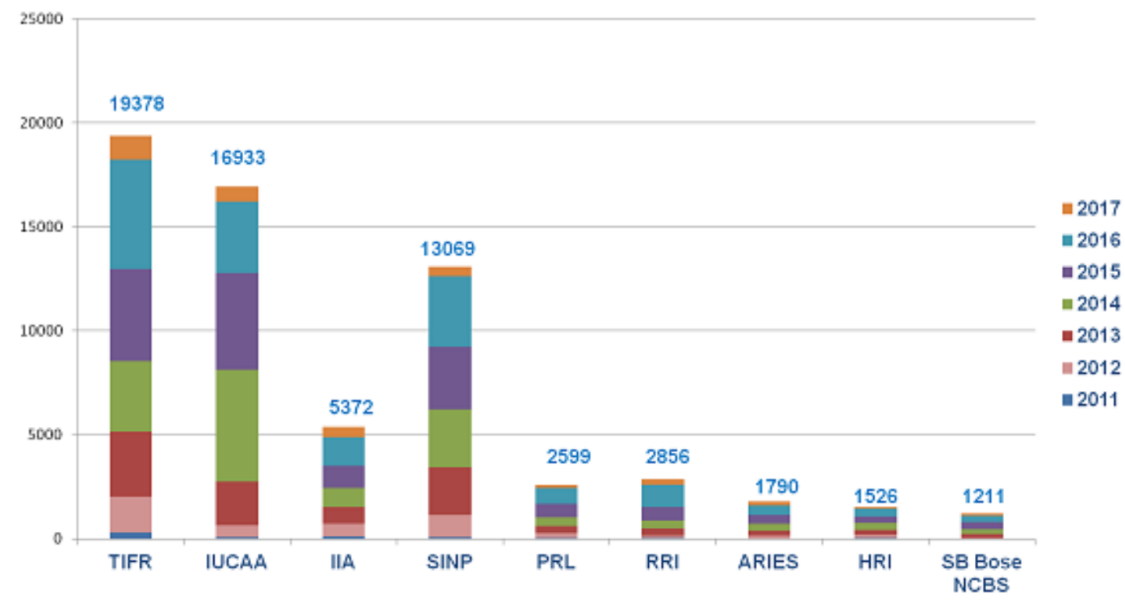

Figure 3. FORSA Institute's Citations

In 2011-15 TIFR received the maximum citations as well. The average citations for TIFR is 2768.28 per year. IUCAA jumped to second in terms of paper publications and its papers received 2418.86 average citations per year.

\section{Conclusion}

It is found that astronomy research papers publication has never had a precise pattern. In comparison to 1991-95 studies, output has been increased three fold in 2011-15. Although this study finds a decline in the number of research output during 2011-2015 for Indian Astronomers, citations have consistently increased every year. Tata Institute of Fundamental Research, Mumbai is the leading institute and IUCAA is an emerging Institute in terms of number of articles published and citations in the field of astronomy and astrophysics research in Indian. It was found that there is a decline in research output during 2011-2015 for Indian astronomers but FORSA institutes have shown progress. Indian studies in the area of astronomy are more collaborative than individual studies. Indian researchers are making contributions in some exorbitantly large international collaborative research projects. Indian researchers prefer to publish their research papers in international journals with the highest impact factors. To conclude, it is clear that Indian astronomy research has a bright future.

\section{References}

[1] Forum for Resource Sharing in Astronomy and Astrophysis http://www.rri.res.in/htmls/library/ forsa.html

[2] N. Anilkumar, Scientometric Study of Doctoral Theses of the Physical Research Laboratory, in 21 st Century Astronomy Librarianship, From New Ideas to Action, edited by Eva Isaksson, Jill Lagerstrom, András Holl, and Nirupama Bawdekar (2010), Vol. 433 of Astronomical Society of the Pacific Conference Series, p. 102-108

[3] S. Barve, Gopal-Krishna, nalysis of the Publication Pattern of Radio Astronomers from India during 1990-2001 in Library an Information Services in Astronomy IV (LISA IV - Prague) edited by B.G. Corbin, E.P. Bryson, M. Wolf, (2003), p. 298-301 
[4] B.M. Meera, M. Manjunath, Productivity of Indian Telescopes: Impact Analysis through Scientometric Methods in Open Science at the Frontiers of Librarianship, edited by András Holl, Soizick Lesteven, Dianne Dietrich, and Antonella Gasperini (2015), Vol. 492 of Astronomical Society of the Pacific Conference Series, p. 118-122

[5] Dwivedi, International Journal of Research in Library Science, 2(3), 155(2016)

[6] V.K. Rai, K.P.S. Senger, Astronomy Research in IUCAA: A Scientometric Study of the Papers Published by IUCAA Academics during 1993-2013 in Open Science at the Frontiers of Librarianship, edited by András Holl, Soizick Lesteven, Dianne Dietrich, and Antonella Gasperini (2015), Vol. 492 of Astronomical Society of the Pacific Conference Series, p. 128-132

[7] V.K. Rai, K.P.S. Senger, S.K. Pathak, A Scientometric Study of Research Papers Published by Visiting Associates of IUCAA, Pune, India during 2003-2013 in Open Science at the Frontiers of Librarianship, edited by András Holl, Soizick Lesteven, Dianne Dietrich, and Antonella Gasperini (2015), Vol. 492 of Astronomical Society of the Pacific Conference Series, p. 123-127

[8] B. Dutta, D.S. Rath, Library Philosophy and Practice (e-journal), (2013) https://digitalcommons. unl.edu/libphilprac/996/ 